\title{
In vitro and in vivo study of commercial calcium phosphate cement HydroSet $^{\mathrm{TM}}$
}

\author{
Niall W. Kent, ${ }^{1,2,3}$ Gordon Blunn, ${ }^{4}$ Natalia Karpukhina, ${ }^{1}$ Graham Davis, ${ }^{1}$ Roberta Ferro de Godoy, ${ }^{4}$ \\ Rory M. Wilson, ${ }^{5}$ Melanie Coathup, ${ }^{4}$ Lyris Onwordi, ${ }^{4}$ Wen Yu Quak, ${ }^{4}$ Robert Hill ${ }^{1}$ \\ ${ }^{1}$ Dental Physical Sciences, Institute of Dentistry, Barts and The London School of Medicine and Dentistry, Queen Mary \\ University of London, London E1 4NS, UK \\ ${ }^{2}$ Centre for Nature Inspired Engineering, University College London, Torrington Place, London, WC1E 7JE, UK \\ ${ }^{3}$ Warwick Medical School, University of Warwick, Coventry, CV4 7AL, UK \\ ${ }^{4}$ John Scales Centre for Biomedical Engineering, Institute of Orthopaedics and Musculoskeletal Science, University College \\ London, Royal National Orthopaedic Hospital, Stanmore, UK \\ ${ }^{5}$ School of Engineering and Materials Science, Queen Mary University of London, London E1 4NS, UK
}

Received 11 July 2016; revised 13 October 2016; accepted 16 October 2016

Published online 7 November 2016 in Wiley Online Library (wileyonlinelibrary.com). DOI: 10.1002/jbm.b.33809

Abstract: The commercial calcium phosphate cement,
HydroSet ${ }^{\mathrm{TM}}$, was investigated in vitro, studying phase forma-
tion, compressive strength and setting time, followed by an
ovine in vivo study to measure osseointegration, bone appo-
sition and bone-to-graft contact. The X-ray diffraction and
${ }^{31} \mathrm{P}$ Magic Angle Spinning Nuclear Magnetic Resonance
(MAS NMR) results showed the initial formation of octacal-
cium phosphate and hydroxyapatite at one hour. Over 7 days
the octacalcium phosphate transformed to apatite, which was
the only crystalline phase of the cement at 28 days. This apa-
tite phase is thought to be a calcium deficient apatite. In the
scanning electron microscopy, histological images of
12-week ovine in vivo results showed a high degree of
osseointegration, $92.5 \%$. Compressive strength comparisons

between in vitro and in vivo measurements showed a dramatic difference between the in vitro measurements (highest 25.4 $\mathrm{MPa}$ ) and in vivo (95 $\mathrm{MPa}$ ), attributed to bone ingrowth into the cement in vivo. To the best of our knowledge this is the first time phase evolution of HydroSet ${ }^{\mathrm{TM}}$ and the properties studied in vitro complement the in vivo evaluation of the cement in a publication. The significance of the new finding of initial formation of octacalcium phosphate in this cement is discussed. $\odot 2016$ Wiley Periodicals, Inc. J Biomed Mater Res Part B: Appl Biomater, 106B: 21-30, 2018.

Key Words: calcium phosphate cement, HydroSet $^{\mathrm{TM}}$, octacalcium phosphate, hydroxyapatite, in vivo, in vitro

How to cite this article: Kent NW, Blunn G, Karpukhina N, Davis G, de Godoy RF, Wilson RM, Coathup M, Onwordi L, Quak WY, Hill R. 2018. In vitro and in vivo study of commercial calcium phosphate cement HydroSet ${ }^{\mathrm{TM}}$. J Biomed Mater Res Part B 2018:106B:21-30.

\section{INTRODUCTION}

Calcium Phosphate Cements, CPCs, are calcium phosphate based synthetic bone graft materials, which form as a result of the reactions between calcium phosphate salts. ${ }^{1,2}$ On mixing with water the salts react to form apatite, ${ }^{3}$ the mineral phase of bone and teeth., ${ }^{4,5}$ CPCs have uses in both orthopaedics and dentistry where they are used as bone substitute materials to replace missing or diseased bone. ${ }^{6}$ They are advantageous because they allow the surgeon to inject the setting cement paste into the implantation site using minimally invasive techniques. ${ }^{7,8}$ These materials are also in vivo setting which allows them to mould to the shape of the implantation site once implanted, which ensures complete void filling. ${ }^{9,10}$ Unlike CPCs, granular bone substitutes do not have this advantage and cannot set in vivo.

The first commercial calcium phosphate cement was developed by Chow in $1983 .^{2}$ In the first patent on calcium phosphate cements tetracalcium phosphate $\left(\mathrm{Ca}_{4}\left(\mathrm{PO}_{4}\right)_{2} \mathrm{O}\right.$, TTCP) and dicalcium phosphate anhydrous $\left(\mathrm{CaHPO}_{4}, \mathrm{DCPA}\right)$ (or dicalcium phosphate di-hydrous $\left(\mathrm{CaHPO}_{4} \cdot 2 \mathrm{H}_{2} \mathrm{O}, \mathrm{DCPD}\right.$ ) were used as the initial reagents. The authors discovered that leaving these mixtures in test tubes led to a 'hard' deposit after a few hours. These calcium phosphate salts are more soluble at neutral $\mathrm{pH}$ than hydroxyapatite $\left(\mathrm{Ca}_{10}\left(\mathrm{PO}_{4}\right)_{6}(\mathrm{OH})_{2}\right)^{11}$ and when mixed with water produce hydroxyapatite. As a consequence of this discovery various animal studies were conducted ${ }^{12-14}$ which demonstrated precipitation of nano-crystalline hydroxyapatite in these compositions. On implantation highly biocompatible hydroxyapatite was readily replaced by new bone. ${ }^{14}$ This cement composition using TTCP and DCPA (or DCPD) was approved by the FDA in 1996 and became the first commercial clinically available calcium phosphate cement. ${ }^{3}$ 
These cements have varied medical applications and are specifically useful in orthopaedic surgery due to the compositional similarity to bone. They are implanted into bone in many procedures; they are used in craniofacial surgery, skeletal fractures, hip replacements, vertebroplasty, kyphoplasty, and in other corrective and restorative orthopaedic procedures. ${ }^{15-18}$

Typical cement setting occurs via a dissolution/precipitation route, ${ }^{1}$ whereby the two calcium phosphate salts dissolve into the aqueous solution to their constituent $\mathrm{Ca}^{2+}$ and $\mathrm{PO}_{4}^{3-}$ ions. These ions then, precipitate to give hydroxyapatite. HydroSet ${ }^{\mathrm{TM}}$ is an apatite based calcium phosphate cement consisting of a powder and solution, that are mixed together to initiate the setting reaction. The powder contains TTCP (73\%) and DCPD (27\%) and the solution $\mathrm{H}_{2} \mathrm{O}$, $\mathrm{Na}_{2} \mathrm{HPO}_{4}, \mathrm{NaH}_{2} \mathrm{PO}_{4}$ and Polyvinylpyrrolidone (PVP). To the authors knowledge no previous studies of in vitro phase evolution of HydroSet ${ }^{\mathrm{TM}}$ have been published. Whilst the combination TTCP and DCPD have been studied extensively HydroSet $^{\mathrm{TM}}$ also contains poly(vinylpyrrolidone) in its formulation, which could potentially affect phase evolution. Hannink et al. studied the phase evolution in vivo and found the formation of hydroxyapatite within the cement; it was also found that some starting powder remained unreacted within the implant for up to 28 weeks. ${ }^{19}$

The aim of this study was to investigate the in vitro and in vivo properties of the commercial calcium phosphate cement HydroSet ${ }^{\mathrm{TM}}$ including the structural characterization combining the XRD and ${ }^{31} \mathrm{P}$ MAS NMR. The latter allows probing the local environment of the phosphorus atoms in solids and delivers the detailed information, regardless whether the environment is crystalline or amorphous. The ${ }^{31} \mathrm{P}$ MAS NMR has been shown to be specifically useful for the identification of octacalcium phosphate $\left(\mathrm{Ca}_{8} \mathrm{H}_{2}(-\right.$ $\left.\mathrm{PO}_{4}\right)_{6} .5 \mathrm{H}_{2} \mathrm{O}$, OCP). ${ }^{20,21}$ Tseng, Mou ${ }^{22}$ previously shown the ability of ${ }^{31} \mathrm{P}$ MAS NMR to successfully differentiate between octacalcium phosphate and hydroxyapatite, which allowed the transition between the two phases to be studied. An advantage of the ${ }^{31} \mathrm{P}$ MAS NMR probe is high sensitivity that allows the detection any of phosphorus containing species present in small amounts. The in vitro aspect was to study phase and mechanical evolution over 28 days of immersion in Tris buffer solution. Using an ovine femoral implant model we aimed to measure osseointegration, bone apposition and compressive strength. The ovine model was used because bone apposition rates better match those found in humans than the rat or rabbit model and the approximate size and weight is similar as to that in humans. $^{23}$

\section{MATERIALS AND METHODS}

\section{In vitro preparation}

HydroSet $^{\mathrm{TM}}$ was mixed according to the manufacturer's instructions to produce a paste. This cement paste was filled into cylindrical steel moulds with a height of $6 \mathrm{~mm}$ and diameter of $4 \mathrm{~mm}$. The cylinders were "over-filled" and sandwiched between two steel plates. The moulds and plates were then clamped together using a G clamp. The clamp was then placed into a $37^{\circ} \mathrm{C}$ oven (Borolabs incubator, Borolabs Basingstoke, UK) for $120 \mathrm{~min}$. The clamp and plates were then removed and the cements, whilst in the moulds were ground with silicon-carbide article to ensure a flat surface at each end of the cylinder.

Each cylinder was then immersed in $10 \mathrm{~mL}$ of Tris buffer solution, as prepared previously, ${ }^{24}$ (in $15 \mathrm{~mL}$ plastic centrifuge tubes) for 1 h, 1 day, 7 days, or 28 days. Eight cement cylinders were immersed at each time point for each cement composition for compressive strength measurements. The samples were stored in a $37^{\circ} \mathrm{C}$ oven for their respective time-points. After being stored each cylinder was removed and patted dry and the compressive strength measured immediately.

\section{Cement property characterization}

Compressive strength measurements. Compressive strength was measured using an Instron 5567 materials property testing machine (Instron, High Wycome, UK) using a $1 \mathrm{kN}$ load cell. Eight cylindrical specimen after their respective immersion periods were placed between two steel plates without drying with a $1 \mathrm{~cm}$ square damp filter article above and below the specimen. Force was applied at a displacement rate of $1 \mathrm{~mm} / \mathrm{min}$. The test was stopped either automatically by the machine when fracture was detected or when it was evident that fracture had occurred.

Setting time measurement. The setting time of each cement sample was measured, in duplicates, using the Gilmore needle test according to the ISO standard ISO (99171:2007(E)). The HydroSet ${ }^{\mathrm{TM}}$ Cement pastes were prepared according to the manufacturer's instructions. The cement paste was filled into cylindrical moulds (Height $4 \mathrm{~mm}$, Diameter $8 \mathrm{~mm}$ ) using a spatula. To measure the initial setting time the 'lighter' Gilmore needle $(2.12 \mathrm{~mm}$ dia. $113.4 \mathrm{~g}$ Wt.) was lowered onto the cement surface and left for 5 seconds, the setting time was taken until the needle tip no longer made an indentation on the cement surface. To measure the final setting time, the 'larger' Gilmore needle (1.06 mm dia. $453.6 \mathrm{~g} \mathrm{Wt}$.) was used and again the needle was lowered onto the surface of the cement paste and left for 5 seconds, the final setting time was taken when the needle no longer made an indentation on the cement surface.

\section{Cement phase analysis}

$X$-ray powder diffraction. Powder XRD experiments for the cements were run on X'Pert Pro PANalytical diffractometer with Bragg Brentano $\theta / \theta$ geometry with $\mathrm{Cu} \mathrm{K} \alpha$ radiation operating at $45 \mathrm{kV}$ and $40 \mathrm{~mA}$. Data were collected from 3 to $702 \theta^{\circ}$ in increments of $0.0334^{\circ}$. Beam divergence was set to $1 / 4^{\circ}$. An X'Celerator RTMS detector was used and the data collection time at each point was equivalent to 200 seconds. The samples were mounted on zero background silicon single crystal substrates.

Magic angle spinning nuclear magnetic resonance. The ${ }^{31} \mathrm{P}$ MAS NMR experiments were run on a Bruker $600 \mathrm{MHz}$ 
spectrometer at the $242.9 \mathrm{MHz}$ resonance frequency. The powder samples were packed into $4 \mathrm{~mm}$ rotor and spun at 11-12 kHz. The measurements were made using 60s recycle delay and $85 \% \mathrm{H}_{3} \mathrm{PO}_{4}$ was used to reference the chemical shift scale.

\section{Surgery and test material administration}

All relevant licenses and ethics approval were in place and procedures carried out in compliance with the UK Home Office as stated in the Animal Scientific Procedures Act (1986). Prior to surgery each sheep was administered the pre-med Rompun $^{\mathrm{TM}}$ [xylazine] and anaesthesia was induced by intravenous administration of ketamine and midazolam and then maintained using isoflurane and oxygen. The anaesthetised animal was prepared for surgery and the medial femoral condyle was exposed by a medial approach. Two holes were drilled into the cancellous bone, one in a proximal position and one in a distal position with $>4 \mathrm{~mm}$ between the holes. The holes were drilled to a standard depth of $15 \mathrm{~mm}$ made with an $8.0 \mathrm{~mm}$ diameter drill bit. $1 \mathrm{~mm}$ holes were drilled either side of the defect and $1 \mathrm{~mm}$ tantalum beads were inserted in order to correctly locate the defects on retrieval using radiography. After irrigating with sterile saline, the test material was pressed into place. The wound was closed and the contra-lateral medial femoral condyle exposed by a medial approach. In a similar manner two holes were drilled, irrigated with sterile saline, test materials inserted and the wound closed. A total of six animals were used with each animal having one defect filled with HydroSet ${ }^{\mathrm{TM}}$.

At the time of surgery, an antibiotic injection of Cepor$\mathrm{ex}^{\mathrm{TM}}$ [cephalexin] was given intramuscularly at a dose of $5 \mathrm{~mL}$ per animal. Prior to recovery from anaesthesia all animals received an analgesic injection of Vetergesic ${ }^{\mathrm{TM}}[2 \mathrm{~mL}$; $0.6 \mathrm{mg}$ Buprenorpine] administered as an intra-muscular injection. Ceporex ${ }^{\text {TM }}$ injections were also given postoperatively for 3 days. Vetergesic ${ }^{\mathrm{TM}}$ was administered daily on the three successive days post-surgery in all animals.

Bone marking fluorochrome injections. The animals received their first bone marking injection on day $63 \pm 2$ (9 weeks) followed by a second bone marking injection 14 days later. The first bone marking injection was an intravenous administration of Alamycin 10 [oxytetracycline] at a dose of $15 \mathrm{mg} / \mathrm{kg}$ body weight and the other an intravenous administration of Calcein green at a dose of $10 \mathrm{mg} / \mathrm{kg}$ body weight.

Terminal procedures. The animals were sacrificed at 12 weeks post-surgery. All animals were euthanized by an overdose of pentobarbital. After sacrifice the femoral condyles including a small part of the femoral shaft were removed from the animal. Samples were radiographed and longitudinal sections were made (with reference to the axis of the defect into which the cement was inserted) through each defect using a diamond band saw. This produced a small slab with an exposed transverse cut face of the implant which was surrounded by at least $5 \mathrm{~mm}$ of bone. One half of each sample was used for mechanical testing and immersed in phosphate buffered saline. Samples were compression tested on the day of sacrifice. Specimens for

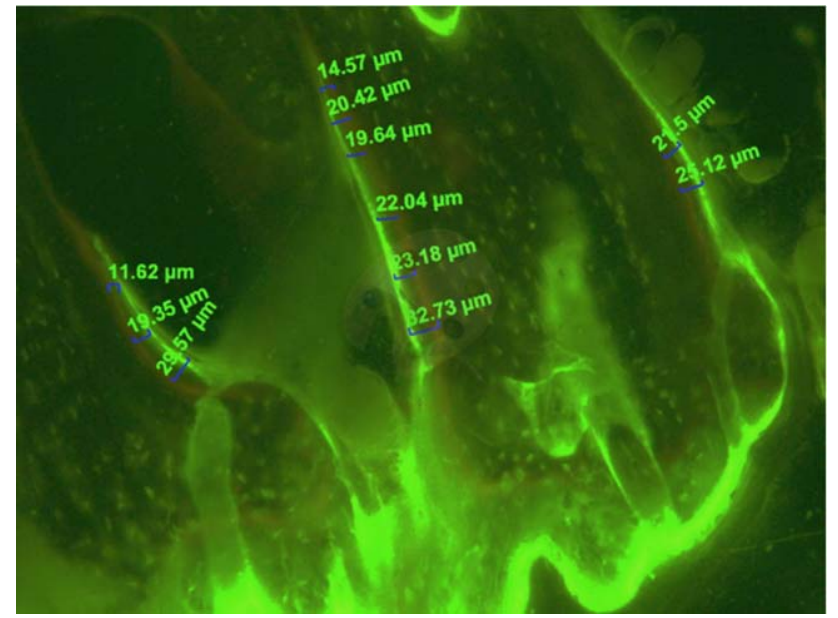

FIGURE 1. Fluorescence microscopy of specimen

histology (and following mechanical testing) were immediately fixed in at least 4 times the tissue volume of $10 \%$ neutral buffered formal saline.

\section{Laboratory phase}

Histological processing and analysis. Each sample was dehydrated in serial dilutions of alcohol, followed by three changes in absolute alcohol before immersing in chloroform to de-fat the specimens. Specimens were returned to absolute alcohol before being immersed in a 50:50 solution of methyl methacrylate resin and absolute alcohol. Samples were transferred to pure methyl methacrylate solution prior to being polymerized in acrylic resin. After embedding, the implant and the bone were sectioned using a band saw. Sections were taken longitudinally through the centre of the implant in the coronal plane. Thin slices (100-60 $\mu \mathrm{m})$ were then prepared using an Exakt saw (Exakt, Norderstedt, Germany).

Bone apposition rates adjacent to the defect surface and within the defects were determined in these thin sections (100-60 $\mu \mathrm{m})$ before staining. This was achieved by measuring the distance between bone markers (Figure 1) under a fluorescence microscope and using image analysis system (Axiovision 4.5; Carl Zeiss, Imaging Associates Ltd, UK).

In all animals, bone turnover rates were calculated from regions along the length of the defect and resulted in a minimum of seven regions of interest being measured per slide. Measurements were taken using the $\times 5$ objective lens. Apposition rates were calculated in $\mu \mathrm{m}_{\text {day }}{ }^{-1}$.

Following fluorochrome marker measurement and analysis, thin sections were stained with Toluidine Blue for 15 min and Paragon for $10 \mathrm{~min}$ and the amount of new bone, bone-to-graft contact and test material present within the defect assessed using an image analysis system (Axiovision 4.5; Carl Zeiss, Imaging Associates Ltd, UK).

In addition to quantifying the distance between the two bone markers, image analysis was also used to quantify the amount of bone-cement contact. This involved capturing images along the entire bone-cement interface using a $\times 10$ 


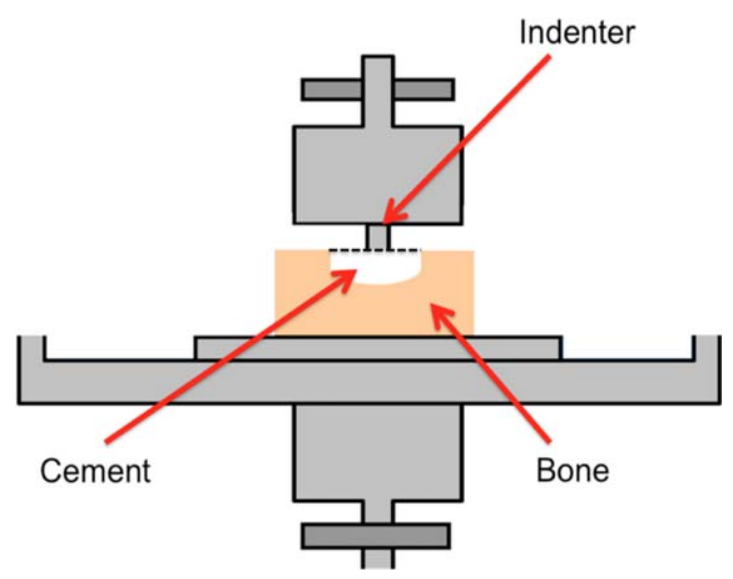

FIGURE 2. Test jig configuration for compression testing.

objective lens and a digital colour camera on the image analysis system. Assessments were used to evaluate the proportion of bone and soft tissue in two dimensions using a line intercept method.

Backscattered scanning electron microscopy. Sections from each test group and at both time-points were sputtercoated and prepared for viewing using JSM 550LV SEM (JEOL, Welwyn Garden City, Hertfordshire, UK).

$X$-ray microtomography. The embedded specimens were scanned using the MuCAT 2 X-ray microtomography (XMT) scanner, designed and run at Queen Mary University of London. $^{25}$ The scanner was operated at $90 \mathrm{kV}, 180 \mu \mathrm{A}$ with a voxel size of $25 \mu \mathrm{m}$. The effective detector size was $1800 \times$ 800 pixels (the width could be adjusted according to specimen size up to 2700 pixels). Effective exposure time was $18 \mathrm{~s}$ for 1901 projections. Tomographic reconstruction was performed using standard cone-beam back-projection.

Mechanical testing. The compressive strength of each of the cements was tested using an indentation method in two central positions (at least $2 \mathrm{~mm}$ apart) within the cement

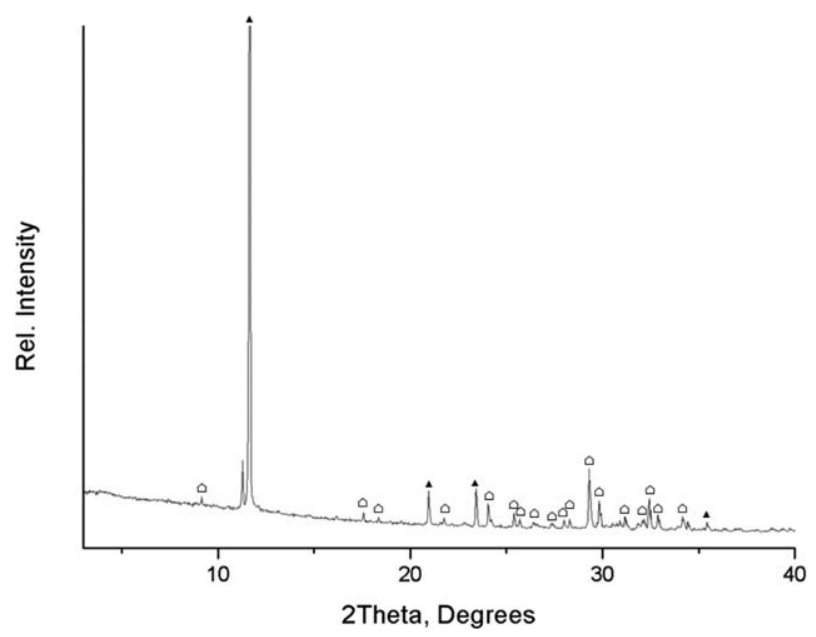

FIGURE 3. XRD of the starting powder.

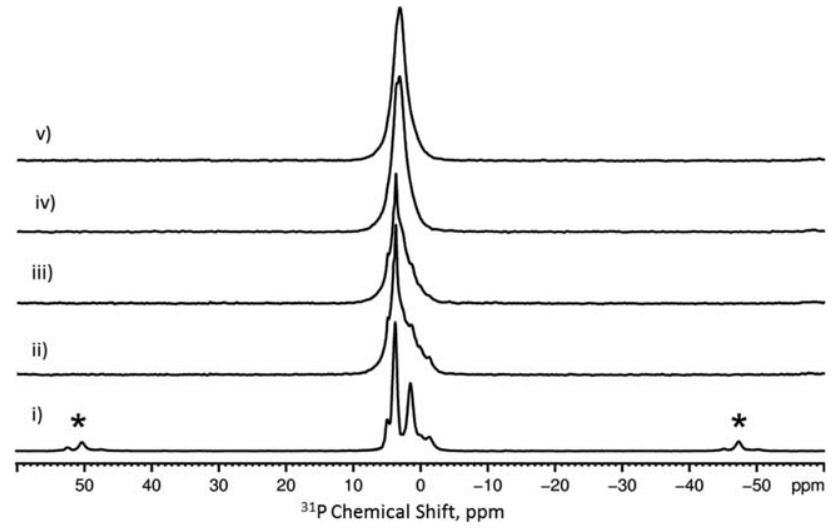

FIGURE 4. ${ }^{31} \mathrm{P}$ MAS NMR spectra for the starting powder (i) and cements matured for (ii) 1 hour, (iii) 1 day, (iv) 7 days and (v) 28 days of immersion in Tris buffer. The asterisks show spinning side bands.

mantle. Testing was performed on a screw driven Zwick Proline 500 test machine fitted with a $500 \mathrm{~N}$ load cell with an accuracy of $0.5 \mathrm{~N}$. Compression testing was performed using a custom-made jig (Figure 2).

The indenter was cylindrical and measured $3 \mathrm{~mm}$ in diameter and was positioned in the center of the test specimen. Before testing could begin, the upper crosshead platen was lowered so as to just make contact with the specimen. In practice this was achieved by applying a preload of $2 \mathrm{~N}$. Load was applied axially to the specimens with a crosshead speed of $5 \mathrm{~mm} / \mathrm{min}$ Specimens were loaded up to yield and beyond into the collapse plateau region of behavior, as defined by Gibson ${ }^{26}$ for elastic-brittle foams. The compressive strength (in MPa) applied to the sample throughout the test was calculated using Eq. (1.1).

$$
\text { Compressive Stress }=\frac{\text { Applied Load, in } \mathrm{N}}{\pi(\text { radius of indenter, in } \mathrm{mm})^{2}}
$$

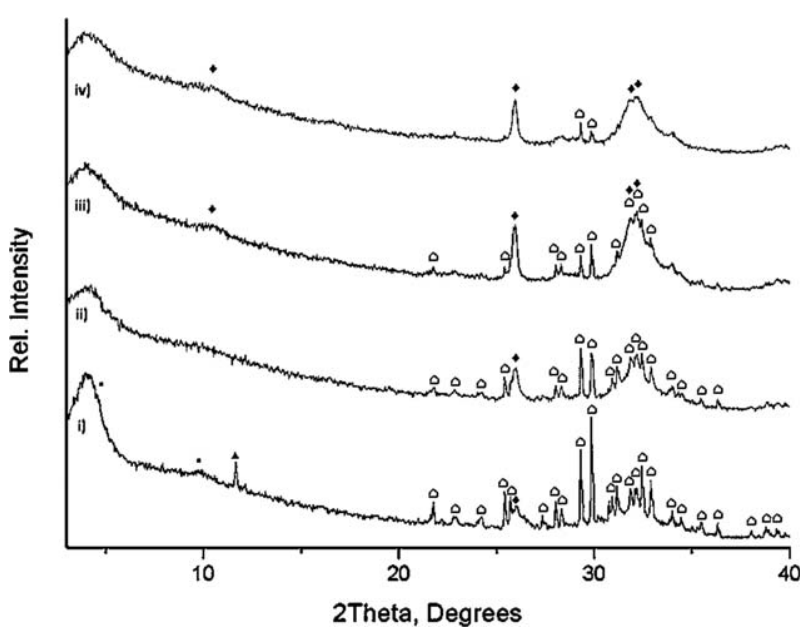

FIGURE 5. XRD patterns of the cements matured for (i) 1 hour, (ii) 1 day, (iii) 7 days and (iv) 28 days of immersion in Tris buffer. The open symbols are for tetracalcium phosphate; the triangles are for the DCPD; squares for the OCP and the diamonds for the apatite. 
TABLE I. Compressive Strength of HydroSet ${ }^{\mathrm{TM}}$ Immersed in Tris buffer Solution at 1 h, 1 Day, 7 Days, and 28 Days

\begin{tabular}{lccc}
\hline \multicolumn{4}{l}{ Compressive Strength $(\mathrm{MPa})$} \\
\hline 1 Hour & 1 Day & 7 Days & 28 Days \\
\hline $14.2( \pm 1.4)$ & $25.4( \pm 2.2)$ & $13.6( \pm 1.8)$ & $25.1( \pm 3.5)$ \\
\hline
\end{tabular}

\section{RESULTS}

\section{In vitro studies}

The X-ray diffraction of the starting material in Figure 3 shows that the phases present are DCPD, tetracalcium phosphate and DCPA. The ${ }^{31} \mathrm{P}$ MAS NMR of the starting material, the bottom spectrum in Figure 4, confirms this. At $1 \mathrm{~h}$ in Tris buffer solution the XRD (Figure 5) shows the presence of DCPD, tetracalcium phosphate and octacalcium phosphate. The latter was identified on X-ray diffraction from low intensity lines at 4.7 and 9.8 degrees two theta. The Xray diffraction shows that in the 1 day, 7 day and 28 day samples two phases are present, tetracalcium phosphate and hydroxyapatite. The only apparent change is the relative intensities of the two phases; the tetracalcium phosphate decreases in relative intensity whereas the hydroxyapatite increases in its relative intensity.
The ${ }^{31} \mathrm{P}$ MAS NMR results (Figure 4 ) show that at 1 hour tetracalcium phosphate is present with resonances at 3.8 and $5.0 \mathrm{ppm}$. There is a broad shoulder at $2.8 \mathrm{ppm}$ suggesting hydroxyapatite is present, as well as a resonance at $1.4 \mathrm{ppm}$ indicating a small fraction of DCPD. There are also several resonances at 3.6, 2.0 and $-0.2 \mathrm{ppm}$ showing that OCP is present. The ${ }^{31} \mathrm{P}$ MAS NMR results show tetracalcium phosphate is still present up to 1 day of the immersion, as can be identified by the resonances at 3.8 and $4.8 \mathrm{ppm}$. This is true also for DCPD where a resonance at $1.4 \mathrm{ppm}$ is present. The resonances at -1.4 and -0.2 ppm show a small fraction of DCPA is still present in the cement phase from the starting materials The DCPA was identified only on the ${ }^{31} \mathrm{P}$ MAS NMR and not on the X-ray diffraction by resonances at -1.4 and $-0.2 \mathrm{ppm}$. The 7 day ${ }^{31} \mathrm{P}$ MAS NMR spectrum has a resonance at $3.0 \mathrm{ppm}$ of hydroxyapatite and two additional resonances at 3.7 and $4.9 \mathrm{ppm}$ of tetracalcium phosphate. The 28 day sample has a main resonance at $2.9 \mathrm{ppm}$ (hydroxyapatite), a shoulder at $3.7 \mathrm{ppm}$ and a very small shoulder at $4.9 \mathrm{ppm}$ (tetracalcium phosphate).

HydroSet $^{\text {TMTM }}$ compressive strength values ranged from 13.6 to 25.4 MPa as shown in Table I. The initial setting time of the HydroSet ${ }^{\mathrm{TM}}$ sample was $6 \mathrm{~min}$ and the final setting time was $19 \mathrm{~min}$.
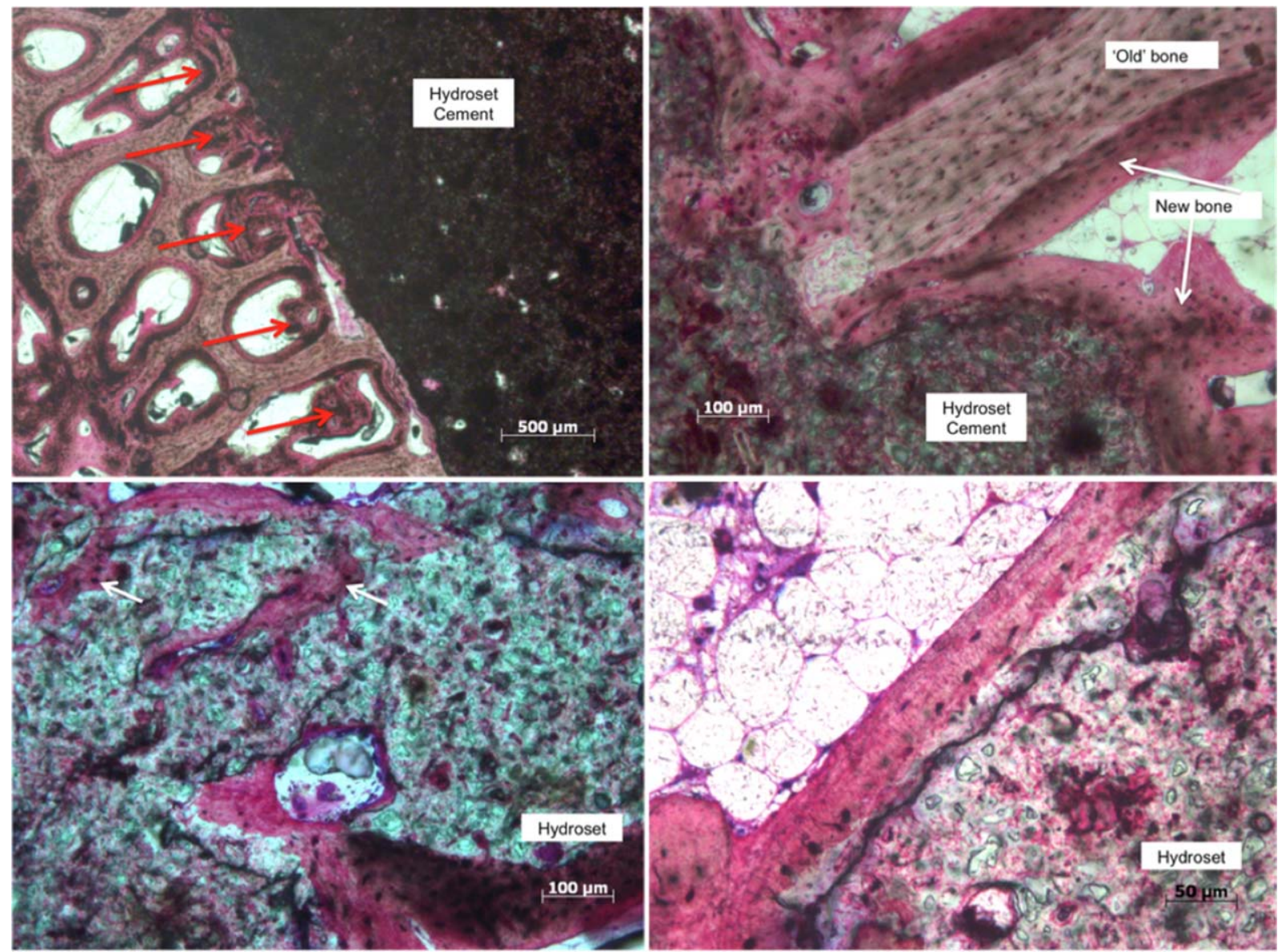

FIGURE 6. Photomicrographs of histological staining showing: Top Left) new bone formation to the bone-cement interface. The red arrows point to clusters of new bone formation within the older trabecular network. Top Right) New bone formation adjacent to the bone-HydroSet ${ }^{\mathrm{TM}}$ interface. Bottom Left) New bone formation within the cement. Bottom Right) New bone formation on the HydroSet ${ }^{\text {TM }}$ surface. 


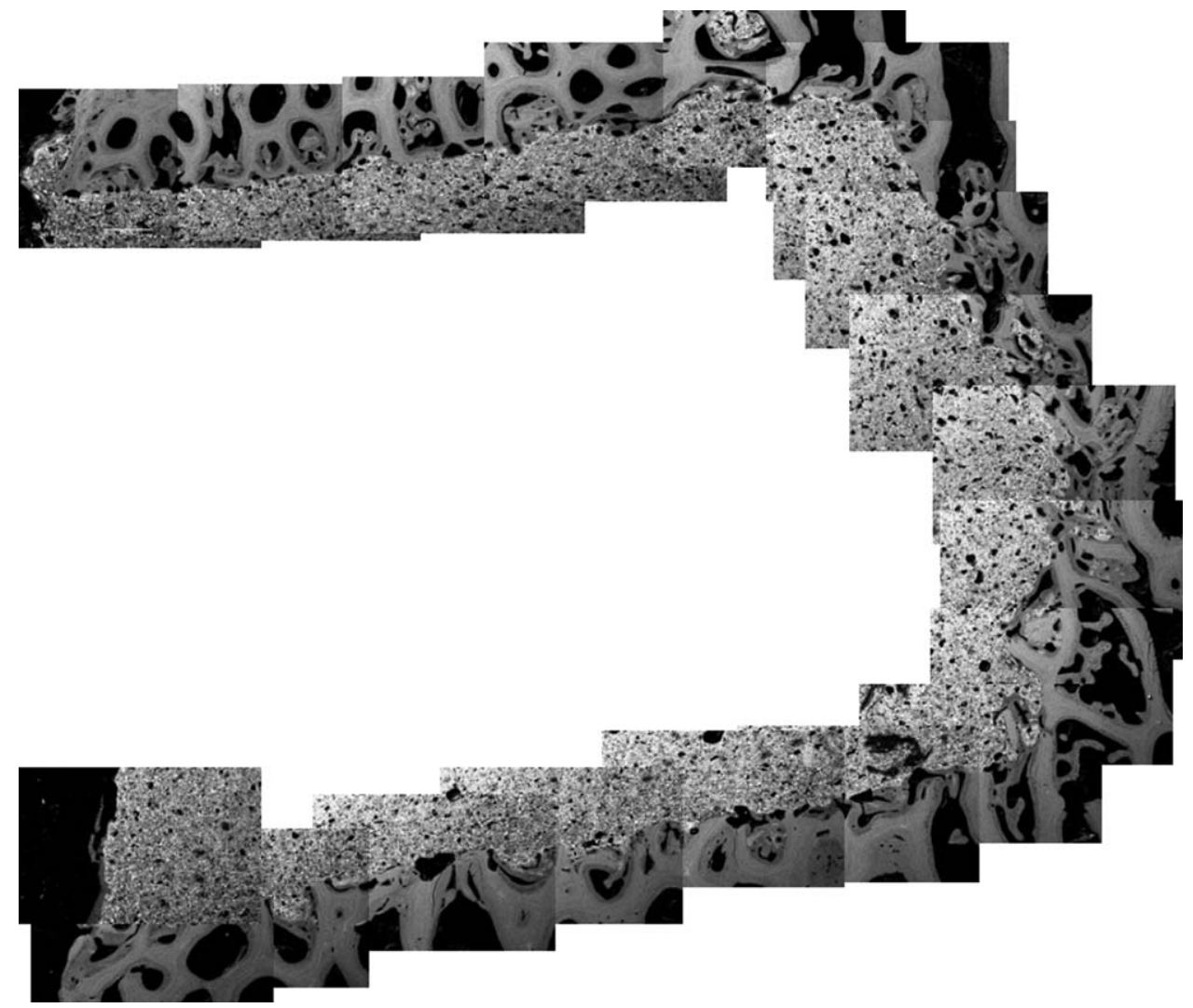

FIGURE 7. A mosaic SEM image showing bone in contact with the cement surface around the length of the defect.

\section{In vivo studies}

Bone apposition rates were measured adjacent to cements in all of the thin sections prepared in this study. The number of measurements made per slide varied according to the distribution of the two markers within the bone, however, a minimal number of seven regions of interest were measured from each slide. Results showed that rate of bone turnover measured was $1.191( \pm 0.345) \mu \mathrm{m} /$ day. Extensive \% bonecement contact was measured in all experimental groups the mean percentage contact being $92.5 \%$. A layer of bone measuring 50-100 $\mu \mathrm{m}$ surrounded almost the entire implant even in region where there was no trabecular contact with the cancellous bone. Bone was also seen with cracks and fissures within the cement (Figures 6-8). In the SEM the cement appeared to be crystalline with bright granules representing TTCP. The cement filled voids within the cancellous bone and this was evidenced by the irregular interface, where the cement had penetrated the soft tissue between trabeculae (Figures 7 and 9). Figure 9 shows an XMT slice of the HydroSet ${ }^{\mathrm{TM}}$ within the implant site twelve weeks after surgery. The slice shows the relatively high radio-opaque HydroSet ${ }^{\mathrm{TM}}$ cement integrated strongly with the trabeculae and disorganised newly formed bone surrounding the cement surface and adjacent trabeculae. The osteoconductive nature of the cement was demonstrated by the bone, which formed over the cement at the opening of the defect site (Figure 9).

Results following mechanical tests showed that the compressive strength of samples in each of the groups was similar with a mean at $95 \mathrm{MPa}$ (Table II). Figures (6 and 7), and 8 show photographs and electron micrographs of the bone/cement interface showing high levels of new bone formation on the cement surface.

Figure 7 shows a mosaic of Back Scattered SEM images overlaid to produce a continuous image of the cement/bone interface. The images show disordered bone formation surrounding both the cement and the original trabecular struts adjacent to the implant site.

\section{DISCUSSION}

Hydroxyapatite was thought to be the only phase that forms during the setting of HydroSet ${ }^{\mathrm{TM}}{ }^{27}$ However, the ${ }^{31} \mathrm{P}$ MAS NMR and XRD results shown here revealed that the cement additionally forms octacalcium phosphate. Strong evidence of OCP on the ${ }^{31} \mathrm{P}$ MAS NMR spectra is a resonance at 3.6 ppm, which would be expected if OCP was present. The other esonances for OCP are at 3.3, 2.0 and -0.2 ppm are partially obscured overlapping with other signals in the narrow range between -1.4 and $5.0 \mathrm{ppm}$. The ${ }^{31} \mathrm{P}$ MAS NMR results are in agreement with the XRD data on OCP as described in the previous section.

Tseng, $\mathrm{Mou}^{22}$ showed the transition from octacalcium phosphate to hydroxyapatite via ${ }^{31} \mathrm{P}$ MAS NMR over $96 \mathrm{~h}$ immersed in solution. The authors showed the initial pure octacalcium phosphate spectrum with resonances at 3.7, 3.3, 2.0 , and $-0.2 \mathrm{ppm}$ and then demonstrated how the resonances at $-0.2,2.0$, and 3.7 ppm gradually decreased over $6 \mathrm{~h}$ 


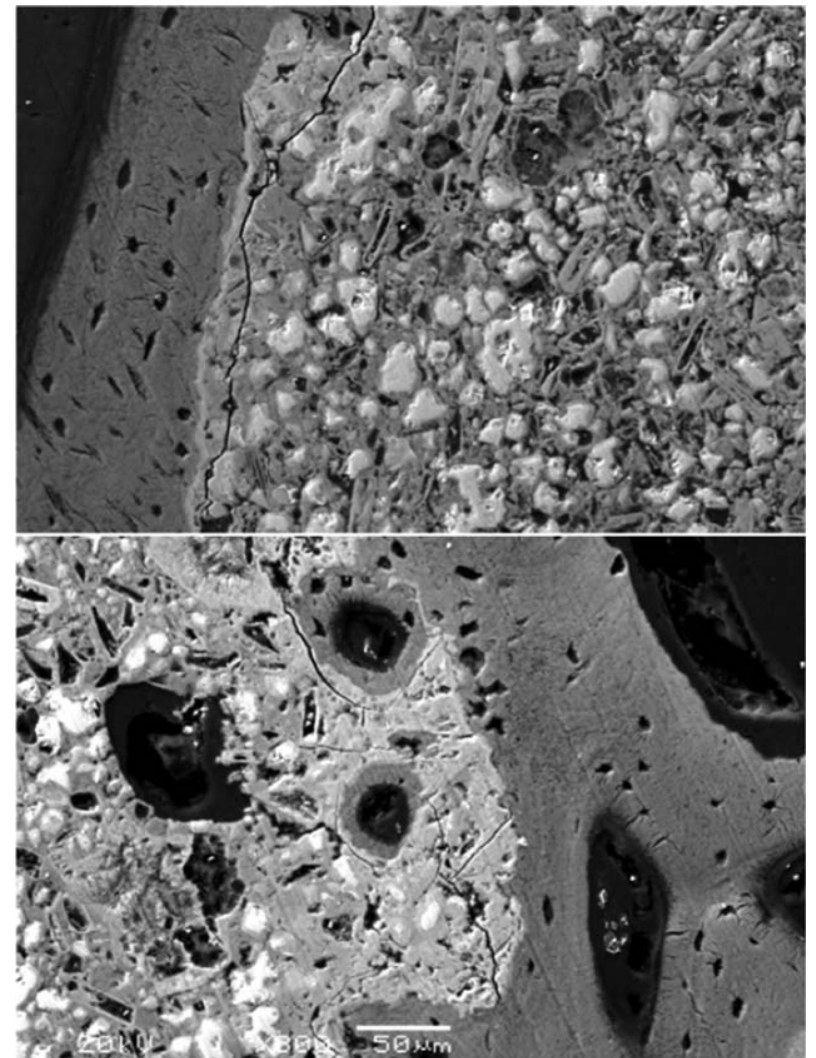

FIGURE 8. SEM Back Scattered micrographs showing bone in direct contact with the cement surface with good integration. Cracks apparent on the SEM images are artefacts and appeared during preparation.

finally reaching relatively low intensity. Eventually only one resonance remained at around $3.0 \mathrm{ppm}$, of hydroxyapatite. The rate of this transition is likely to be affected by the temperature, $\mathrm{pH}$ and ion constituents of the immersion solution.

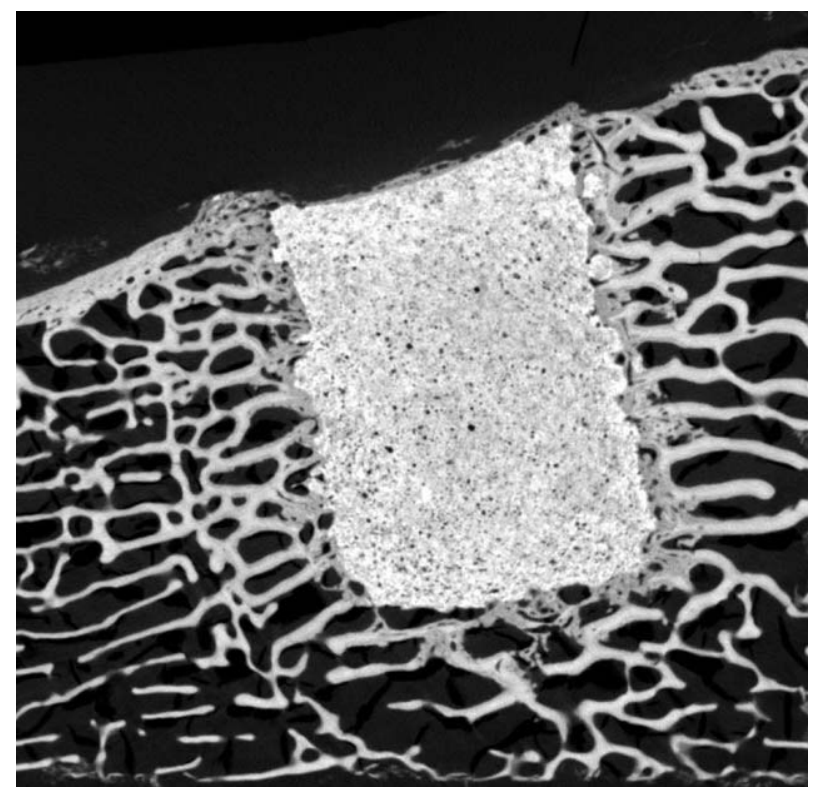

FIGURE 9. X-ray microtomography slice though the implanted HydroSet $^{\mathrm{TM}}$ within bone. The contrast has been increased to highlight differences between new and old bone.
TABLE II. The Minimum, Maximum, and Mean Compressive Strength Values Obtained in Each of the Groups

\begin{tabular}{lccc}
\hline $\begin{array}{l}\text { Minimum } \\
(\mathrm{MPa})\end{array}$ & $\begin{array}{c}\text { Maximum } \\
(\mathrm{MPa})\end{array}$ & $\begin{array}{c}\text { Mean } \\
\text { (MPa) }\end{array}$ & $\begin{array}{c}\text { Std. } \\
\text { Deviation }\end{array}$ \\
\hline 78.4 & 142.2 & 95.0 & 27.2 \\
\hline
\end{tabular}

It is likely that the samples containing octacalcium phosphate in our results are already in the process of transition into an apatite since the spectra shown more closely match the 5 or $6 \mathrm{~h}$ samples of Tseng et al. explaining the lack of relative intensity of the resonances at $-0.2,2.0$, and $3.7 \mathrm{ppm}$.

It is known that OCP often acts as a precursor to hydroxyapatite because OCP nucleates and grows more readily than hydroxyapatite and its formation is kinetically more favourable than that of hydroxyapatite under certain conditions. ${ }^{28}$ Previous studies have shown this occurring in vitro $^{28,29}$ and there have been studies finding OCP during osteogenesis. $^{4,30}$ However, it is not known from these results if the OCP forming is a precursor to the hydroxyapatite formation in this particular cement, or if it forms in addition to the hydroxyapatite.

OCP forming calcium phosphate cements have been reported previously ${ }^{31}$ but this phase was not considered as a precursor species to hydroxyapatite in cements; although this may have been due to difficulties in the identification of the OCP phase. The main reason why no studies have previously found octacalcium phosphate in this setting reaction is probably because typical investigation is restricted to a narrow two theta range during X-ray diffraction analysis. Octacalcium phosphate and hydroxyapatite, due to their similar crystal structures, have similar diffraction patterns. The main difference is a line at 4.7 degrees two theta (with $\mathrm{Cu} \mathrm{K}_{\alpha} \mathrm{X}$-rays) present in only the OCP pattern.

The X-ray diffraction and ${ }^{31} \mathrm{P}$ MAS NMR results show the octacalcium phosphate transforming to hydroxyapatite during the 28 days in Tris buffer solution. Whilst the DCPD fully dissolves between 1 day and 7 days, the tetracalcium phosphate is still present at 28 days.

It is believed that the reaction from OCP to hydroxyapatite can occur through crystal transition as well as through dissolution and re-precipitation. This crystal transition route is supported by a number of studies showing intercrystalline mixtures of OCP and hydroxyapatite. ${ }^{4,32}$ A crystal of partly hydrolyzed OCP when studied on XRD gave the pattern of OCP and hydroxyapatite with the $b$ - and $c$ - axis collinear. ${ }^{4}$ This suggests an intercrystalline mixture of the two crystals given the similarity in their crystal structure. The proposed setting reaction of HydroSet ${ }^{\mathrm{TM}}$ is listed below:

1. $\mathrm{CaHPO}_{4} \cdot 2 \mathrm{H}_{2} \mathrm{O}+2 \mathrm{Ca}_{4}\left(\mathrm{PO}_{4}\right)_{2} \mathrm{O}+\mathrm{H}_{2} \mathrm{O} \rightarrow \mathrm{Ca}_{8}\left(\mathrm{HPO}_{4}\right)_{2}\left(\mathrm{PO}_{4}\right)_{4}$. $5 \mathrm{H}_{2} \mathrm{O}+2 \mathrm{Ca}^{2+}+2(\mathrm{OH})$

2. $\mathrm{Ca}_{8}\left(\mathrm{HPO}_{4}\right)_{2}\left(\mathrm{PO}_{4}\right)_{4} \cdot 5 \mathrm{H}_{2} \mathrm{O}+2 \mathrm{Ca}^{2+}+2(\mathrm{OH})^{-} \rightarrow \mathrm{Ca}_{10}\left(\mathrm{PO}_{4}\right)_{6}$ $(\mathrm{OH})_{2}+2 \mathrm{H}^{+}+5 \mathrm{H}_{2} \mathrm{O}$

The ${ }^{31} \mathrm{P}$ MAS NMR was also very useful for phase identification and the detection of impurities. Whilst the 
formulation of HydroSet $^{\mathrm{TM}}$ is only supposed to contain DCPD and TTCP, the NMR showed the presence of a small fraction of DCPA that was not detected on the X-ray diffraction. Another interesting result is the amount of time the tetracalcium phosphate takes to fully react to form the cement phase. The two starting reagents are added together with an overall calcium to phosphorus ratio of 1.67 to favour apatite formation. ${ }^{27}$ This could be due to the relative particle sizes of the two starting reagents or lack of water penetrating the cement cylinder causing the dissolution of the tetracalcium phosphate and precipitation of the cement phase, as it has been shown that particle size can have a large influence on the reactivity of starting components in calcium phosphate cements. ${ }^{33}$ Hannink et al. showed that the TTCP in the cement remains until twenty six weeks after implantation in vivo. ${ }^{19}$ The scanning electron micrographs (Figure 8) of the implant surface show residual granules within the cement block in the implant site, it is possible that these are remnant unreacted TTCP particles.

Stryker, the manufacturers of HydroSet ${ }^{\mathrm{TM}}$ state that HydroSet $^{\mathrm{TM}}$ has an initial setting time of $4.5 \mathrm{~min}$ and a final setting time of $8.5 \mathrm{~min}^{34}$ whereas the results using the Gilmore needle test give an initial setting time of $6 \mathrm{~min}$ and final setting time of $19 \mathrm{~min}$. This difference is because the manufacturers use a different method to measure the initial and final setting times. This highlights the fact that setting time is a difficult thing to assess and measure, especially when applied to a clinically meaningful context. The assessment should measure whether the material sets well enough in vivo to allow the clinician to easily implant it for a specific application, whilst not causing any negative effects such as washout or disfigurement.

The compressive strength of the cement showed a maximum compressive strength of $25 \mathrm{MPa}$ at one and twentyeight days. Interestingly, a decrease in compressive strength is seen between these two points at seven days to $13 \mathrm{MPa}$, which correlates with and perhaps is caused by the hydrolysis of OCP to hydroxyapatite, identified on the phase analysis. A study performed by Clarkin et al. investigated the mechanical properties of HydroSet ${ }^{\mathrm{TM}}$ and found compressive strengths of between 18 and $24 \mathrm{MPa}$, comparable to this study. ${ }^{27}$ A second study performed by Van Lieshout, Van Kralingen ${ }^{35}$ showed a similar compressive strength of around $11 \mathrm{MPa}$ after three days of storage in water, a similar result to that found here. With respect to the relevance of this to clinical use these levels are approximately similar to vertebral bone ${ }^{27}$ and trabecular bone. ${ }^{36}$

The in vivo results show very high rates of osseointegration for HydroSet ${ }^{\mathrm{TM}}$. The measurement of graft-to-bone contact measured from histological images give a mean percentage contact of $92.5 \%$. This is supported in the microscopy, SEM and X-ray micro-tomography results. The photomicrographs of histological sections show the interface between the cement and original trabecula bone struts. New bone formation is clearly distinguishable from these images, this is highlighted in Figure 6 where new bone is clearly labelled forming on both the cement surface and trabeculae present before surgery. Similarly, the scanning electron micrographs show a strong union between the bone and cement surface. High osseointegration is important for stability of the bone graft within the implant site, which is especially important for these types of materials as they are frequently used to augment metal screws or augment an arthroplasty procedure. With respect to other calcium phosphate based grafting materials, a study by Chan et al. using the same animal model showed bone-to-graft contact of $<50 \%$ for a hydroxyapatite based granular bone substitute implanted for a similar period. ${ }^{37}$

In a study performed by Hannink et al. HydroSet ${ }^{\mathrm{TM}}$ was implanted into the distal femur of rabbits. ${ }^{19}$ The authors measured percentage bone contact between the cement and new bone at 6, 26, and 52 weeks of implantation. Percentage contact was approximately $80-85 \%$ bone-to-graft contact, for all time points measured. The authors also compared Norian SRS and BoneSource in the same study and found similar levels of bone contact for these too. These levels are similar to what was measured here and therefore suggest high levels of osseointegration are associated with calcium phosphate cements. It should be noted that all of the cements studied consist of mixtures of TTCP and DCPD. ${ }^{19}$ A study by Flautre et al. ${ }^{38}$ implanted four compositions of injectable calcium phosphate cement in an distal femur ovine animal model. The authors compared a brushite forming calcium phosphate cement (BCPC), an empty site and defects filled with autologous bone. Although the authors did not directly measure bone-to-graft contact, the microradiographs presented in the article showed very limited osseointegration in the implant site. The authors found bone apposition rates of $1.5 \mu \mathrm{m}$ day $^{-1}$ for the BCPC, 1.2 $\mu \mathrm{m} \mathrm{day}{ }^{-1}$ for the empty site and $1.1 \mu \mathrm{m}$ day $^{-1}$ for the autograft at 24 weeks. The ovine model is believed to accurately represent bone apposition rates compared to that in humans. ${ }^{23}$ The bone apposition rate, in the results presented here, was measured as $1.191( \pm 0.345) \mu \mathrm{m}$ day $^{-1}$, with respect to other bone substitutes this value is comparable to other injectable calcium phosphate cements implanted within sheep.

The considerably higher in vivo compressive strength compared to that measured in vitro was surprising. The highest in vitro compressive strength measurement was 25 MPa whereas in vivo at 12 weeks this value was $94 \mathrm{MPa}$. The cause of this increase is likely due to the ingrowth of bone (shown on the histology) into the cement pores that would have been "unfilled" in vitro. This explanation is supported by the results of Van Lieshout, Van Kralingen ${ }^{35}$ who showed that porosity and compressive strength are negatively correlated. This result also raises questions with respect to the in vitro measurements of bone cements and their validity. Calcium phosphate cements are sometimes dismissed for certain applications due to their relatively low, in vitro, mechanical properties in favor of stronger acrylic or ionomer bone cements. These results suggest that over time in vivo these cements are actually much stronger, due to their ability to quickly osseointegrate, than previously thought. 
One of the most significant aspects of this article is the discovery of the formation of octacalcium phosphate. The HydroSet $^{\mathrm{TM}}$ starting reagents are identical to those originally formulated in CPCs (and are commonly used in commercial examples) it is likely that many other CPC formulations also form OCP but it has not been previously identified. Octacalcium phosphate has been shown to enhance bone formation in vivo, ${ }^{39,40}$ have a positive impact on cell proliferation, ${ }^{41}$ and to be resorbed more readily than hydroxyapatite. ${ }^{42}$

\section{CONCLUSIONS}

The article presented phase evolution, properties and in vivo study of the HydroSet ${ }^{\mathrm{TM}}$ cement. In addition to the apatite phase known to form as a result of the setting, an OCP phase was also found at early stage in the cement. This implies that a number of other cements including commercially available ones that use similar starting calcium phosphates form OCP during the setting. In HydroSet ${ }^{\mathrm{TM}}$, in vitro results showed an initial setting time of 6 minutes, a final setting time of 19 minutes and maximum compressive strength of $25 \mathrm{MPa}$. In vivo results showed bone apposition rates of $1.191( \pm 0.345) \mu \mathrm{m}$ day $^{-1}$, bone-to-graft contact of $92.5 \%$ and an in vivo compressive strength of $95 \mathrm{MPa}$. Comparisons of in vitro and in vivo measurements showed that HydroSet ${ }^{\mathrm{TM}}$ is considerably stronger in vivo compared to in vitro, the cause of this attributed to bone formation within the cement pores and a high degree of osseointegration.

\section{ACKNOWLEDGMENTS}

The authors would like to thank Donal O'Mahony ay Stryker Corp. for supplying the HydroSet ${ }^{\mathrm{TM}}$ samples for this study. The authors would also like to thank Gillian Hughes and Harold Toms for their assistance in performing the in-vivo and ${ }^{31} \mathrm{P}$ MAS NMR studies respectively.

\section{REFERENCES}

1. Dorozhkin SV. Calcium orthophosphate cements and concretes. Adv Condens Matt Mat 2010;7:99-169.

2. Brown WE, Chow LC. A new calcium-phosphate setting cement. J Dent Res 1983;62:672.

3. Chow LC. Development of self-setting calcium-phosphate cements. Nippon Seram Kyo Gak 1991;99:954-964.

4. Elliott J. Structure and Chemistry of the Apatites and Other Calcium Orthophosphates. Amsterdam: Elsevier; 1994.

5. Skinner HCW. Minerals and human health. Emu Notes Mineralog 2013;13:441-484.

6. Claes L, Hoellen I, Ignatius A. Biodegradable bone cements. Orthopade 1997;26:459-462.

7. Khairoun I, Boltong MG, Driessens FCM, Planell JA. Some factors controlling the injectability of calcium phosphate bone cements. J Mater Sci-Mater M 1998;9:425-428.

8. Frayssinet $\mathrm{P}$, Gineste $\mathrm{L}$, Conte $\mathrm{P}$, Fages J, Rouquet N. Short-term implantation effects of a DCPD-based calcium phosphate cement. Biomaterials 1998;19:971-977.

9. Miyamoto $Y$, Ishikawa K, Fukao H, Sawada M, Nagayama M, Kon $M$, et al. In-vivo setting behavior of fast-setting calcium-phosphate cement. Biomaterials 1995;16:855-860.

10. Munting E, Mirtchi AA, Lemaitre J. Bone repair of defects filled with a phosphocalcic hydraulic cement - an in-vivo study. J Mater Sci-Mater M 1993;4:337-344.

11. Hwang JJH, Siew C, Robinson P, Gruninger SE, Chow LC, Brown WE. Functional-evaluation of a new calcium-phosphate cement. J Dent Res 1986;65:195.
12. Costantino PD, Friedman CD, Jones $K$, Chow LC, Sisson GA. Experimental hydroxyapatite cement cranioplasty. Plastic Reconstructive Surg 1992;90:174-185; discussion 86-91.

13. Friedman CD, Costantino PD, Jones K, Chow LC, Pelzer HJ, Sisson GA Sr. Hydroxyapatite cement. II. Obliteration and reconstruction of the cat frontal sinus. Arch Otolaryngol Head Neck Surg 1991;117:385-389.

14. Costantino PD, Friedman CD, Jones K, Chow LC, Pelzer HJ, Sisson GA Sr. Hydroxyapatite cement. I. Basic chemistry and histologic properties. Arch Otolaryngol Head Neck Surg 1991;117: 379-384.

15. Friedman CD, Costantino PD, Takagi S, Chow LC. BoneSource (TM) hydroxyapatite cement: A novel biomaterial for craniofacial skeletal tissue engineering and reconstruction. J Biomed Mater Res 1998;43:428-432.

16. Moore DC, Frankenburg EP, Goulet JA, Goldstein SA. Hip screw augmentation with an in situ setting calcium phosphate cement: An in vitro biomechanical analysis. J Orthop Trauma 1997;11: 577-583.

17. Nakano M, Hirano N, Matsuura K, Watanabe $H$, Kitagawa $H$, Ishihara $\mathrm{H}$, et al. Percutaneous transpedicular vertebroplasty with calcium phosphate cement in the treatment of osteoporotic vertebral compression and burst fractures. J Neurosurg 2002;97:287293.

18. Matsuyama $Y$, Goto $M$, Yoshihara H, Tsuji T, Sakai $Y$, Nakamura $\mathrm{H}$, et al. Vertebral reconstruction with biodegradable calcium phosphate cement in the treatment of osteoporotic vertebral compression fracture using instrumentation. J Spinal Disord Tech 2004;17:291-296.

19. Hannink G, Wolke JGC, Schreurs BW, Buma P. In vivo behavior of a novel injectable calcium phosphate cement compared with two other commercially available calcium phosphate cements. J Biomed Mater Res B 2008;85:478-488.

20. Tseng $\mathrm{YH}$, Zhan JH, Lin KSK, Mou CY, Chan JCC. High resolution P-31 NMR study of octacalcium phosphate. Solid State Nucl Mag 2004;26:99-104.

21. Tsai TWT, Chou FC, Tseng YH, Chan JCC. Solid-state P-31 NMR study of octacalcium phosphate incorporated with succinate. Phys Chem Chem Phys 2010;12:6692-6697.

22. Tseng $Y H$, Mou CY, Chan JCC. Solid-state NMR study of the transformation of octacalcium phosphate to hydroxyapatite: A mechanistic model for central dark line formation. J Am Chem Soc 2006;128:6909-6918.

23. Pearce Al, Richards RG, Milz S, Schneider E, Pearce SG. Animal models for implant biomaterial research in bone: A review. Eur Cells Mater 2007;13:1-10.

24. Chen XJ, Chen XH, Brauer DS, Wilson RM, Hill RG, Karpukhina N. Bioactivity of sodium free fluoride containing glasses and glassceramics. Materials 2014;7:5470-5487.

25. Davis GR, Evershed ANZ, Mills D. Quantitative high contrast Xray microtomography for dental research. J Dent 2013;41:475482.

26. Gibson LJ, Ashby MF. Cellular Solids. Structure and Properties, 2nd ed: Cambridge University Press; 1997.

27. Clarkin OM, Boyd D, Madigan S, Towler MR. Comparison of an experimental bone cement with a commercial control, Hydroset (TM). J Mater Sci-Mater M 2009;20:1563-1570.

28. Johnsson MSA, Nancollas GH. The role of brushite and octacalcium phosphate in apatite formation. Crit Rev Oral Biol M 1992;3: 61-82.

29. Meyer JL, Eanes ED. A thermodynamic analysis of the secondary transition in the spontaneous precipitation of calcium phosphate. Calcif Tissue Res 1978;25:209-216.

30. Brown WE, Eidelman N, Tomazic B. Octacalcium phosphate as a precursor in biomineral formation. Adv Dental Res 1987;1:306313.

31. Honda Y, Anada T, Kamakura S, Morimoto S, Kuriyagawa T, Suzuki O. The effect of microstructure of octacalcium phosphate on the bone regenerative property. Tissue Eng Pt A 2009;15:19651973.

32. Rodriguez-Lorenzo LM. Studies on calcium deficient apatites structure by means of MAS-NMR spectroscopy. J Mater Sci Mater Med 2005;16:393-398. 
33. Ishikawa K, Takagi S, Chow LC, Ishikawa Y. Properties and mechanisms of fast-setting calcium-phosphate cements. J Mater SciMater M 1995;6:528-533.

34. Co SLG. HydroSet: Injectable HA Bone Substitute. Instruction for Use. Kalamazoo: Stryker; 2010. pp 1-4.

35. Van Lieshout EMM, Van Kralingen GH, El-Massoudi Y, Weinans $H$, Patka P. Microstructure and biomechanical characteristics of bone substitutes for trauma and orthopaedic surgery. Bmc Musculoskel Dis 2011;12.

36. Mosekilde L, Kragstrup J, Richards A. Compressive strength, ash weight, and volume of vertebral trabecular bone in experimental fluorosis in pigs. Calcif Tissue Int 1987;40:318-322.

37. Chan O, Coathup MJ, Nesbitt A, Ho CY, Hing KA, Buckland T, et al. The effects of microporosity on osteoinduction of calcium phosphate bone graft substitute biomaterials. Acta Biomater 2012;8:2788-2794.

38. Flautre $B$, Delecourt C, Blary MC, Van Landuyt $P$, Lemaitre $J$ Hardouin P. Volume effect on biological properties of a calcium phosphate hydraulic cement: Experimental study in sheep. Bone 1999;25:35s-9s.

39. Suzuki O, Kamakura S, Katagiri T, Nakamura M, Zhao $\mathrm{BH}$, Honda $Y$, et al. Bone formation enhanced by implanted octacalcium phosphate involving conversion into $\mathrm{Ca}$-deficient hydroxyapatite. Biomaterials 2006;27:2671-2681.

40. Anada T, Kumagai T, Honda Y, Masuda T, Kamijo R, Kamakura S, et al. Dose-dependent osteogenic effect of octacalcium phosphate on mouse bone marrow stromal cells. Tissue Eng Pt A 2008;14: 965-978.

41. Morimoto S, Anada T, Honda Y, Suzuki O. Comparative study on in vitro biocompatibility of synthetic octacalcium phosphate and calcium phosphate ceramics used clinically. Biomed Mater 2012;7.

42. Kamakura S, Sasano Y, Shimizu T, Hatori K, Suzuki O, Kagayama $\mathrm{M}$, et al. Implanted octacalcium phosphate is more resorbable than beta-tricalcium phosphate and hydroxyapatite. J Biomed Mater Res 2002;59:29-34. 